\title{
Entropic Electrokinetics: Recirculation, Particle Separation, and Negative Mobility
}

\author{
Paolo Malgaretti, ${ }^{1, *}$ Ignacio Pagonabarraga, ${ }^{1}$ and J. Miguel Rubi ${ }^{1,2}$ \\ ${ }^{1}$ Department de Fisica Fonamental, Universitat de Barcelona, Carrer Martí i Franqués, 08028-Barcelona, Spain \\ ${ }^{2}$ Department of Chemistry, Imperial College London, SW7 2AZ London, United Kingdom
}

(Received 23 December 2013; published 15 September 2014)

\begin{abstract}
We show that when particles are suspended in an electrolyte confined between corrugated charged surfaces, electrokinetic flows lead to a new set of phenomena such as particle separation, mixing for lowReynolds micro- and nanometric devices, and negative mobility. Our analysis shows that such phenomena arise, for incompressible fluids, due to the interplay between the electrostatic double layer and the corrugated geometrical confinement and that they are magnified when the width of the channel is comparable to the Debye length. Our characterization allows us to understand the physical origin of such phenomena, therefore, shedding light on their possible relevance in a wide variety of situations ranging from nano- and microfluidic devices to biological systems.
\end{abstract}

DOI: 10.1103/PhysRevLett.113.128301

PACS numbers: 82.39.Wj, 47.56.+r, 47.61.Fg

The recent development of nano- and microfluidic devices [1], as well as cellular regulation mechanisms and cellular signaling [2], relies on the transport of ions across channels or pores whose sections range from the nanometric to the micrometric scale [3-5]. The transport across such conduits has been characterized, even for varying-section channels [6-11], assuming that the channel width $h(x)$ is large compared to the Debye length $\kappa^{-1}$ over which the electrolyte charge distributes in the neighborhood of the charged channel wall $[\kappa h(x) \ll 1]$, or in the absence of electrolytes [12]. Nowadays, the continuous process of device miniaturization and the widening of the range of achievable salt concentrations require an understanding of the behavior of such systems when the relevant length scales compete with each other [13]. Such regimes are already exploitable in different micro- and nanofluidic experiments $[3,4]$ and can be relevant in a variety of biological systems $[14,15]$.

In this Letter, we will show that, precisely in this regime, i.e., when the Debye length and the channel aperture are comparable in size, $\kappa h(x) \sim 1$, an electrolyte embedded in a corrugated channel develops new transport regimes that can be exploited to separate suspended particles, control electric and mass currents, and eventually induce negative mobility. When $\kappa h(x) \sim 1$, the electrolyte response to external forcing, such as electrostatic fields, is very sensitive to the channel shape, and it develops a recirculating region in which the electrolyte flows on the opposite direction as compared to the average volume flow, as shown in Fig. 1(a). Such a phenomenon, typical for incompressible fluids, is due to the interplay between the electrostatic double layer and the varying geometrical confinement, and its magnitude is significantly amplified when $\kappa h(x) \sim 1$. We coin this regime entropic electrokinetics since the phenomena we identify can only arise due to the spatially varying constriction induced by the geometrical confinement. This variation affects the local spatial distribution of ions, essentially controlled by the interplay between the wall charge and the ion entropy. We will show that the entropic variations in the charge density
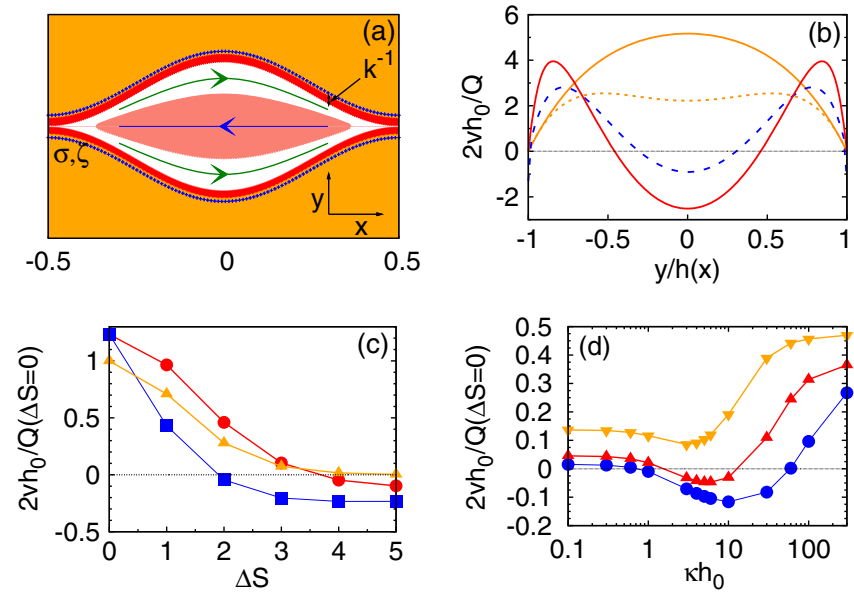

FIG. 1 (color online). Geometry and characteristic flows of entropic electro-osmotic transport. (a) Schematic view of the channel. (b) Velocity profiles for an electrolyte electroosmotically driven inside a varying-section insulating channel of section $h(x)$ (as described in the text), at three positions along the channel, $x / L=0,0.25,0.35,0.4$ (red solid, blue dashed, orange dotted, and red thick, respectively) for $\kappa h_{0}=5$, $\beta z e \Delta V=0.01, \Delta S=3$. (c) Mass flow, $Q /\left(2 h_{0}\right)$ (triangles), average velocity along the channel longitudinal symmetry axis (circles), and velocity at $x / L=1 / 2, y=0$ (squares) normalized by the corresponding mass flow along a uniform section channel, $Q(\Delta S=0)$, as a function of the corrugation for $\kappa h_{0}=5$, $\beta z e \Delta V=0.01$. (d) Average fluid velocity along the longitudinal axis normalized by the corresponding mass flow along a uniform section channel, $Q(\Delta S=0)$, as a function of $\kappa$ for $\beta z e \Delta V=0.01$ and $\Delta S=3$ (orange down triangles), $\Delta S=4$ (red up triangles), $\Delta S=5$ (blue circles). 
induced by the geometric constraints of the corrugated walls have a significant influence in the electrokinetics for narrow channels. Moreover, the dynamics of suspended particles is strongly affected by the local recirculation of the electrolyte: phenomena such as current inversion particle separation and negative mobility can be attained appropriately tuning the channel shape. Therefore, entropic electrokinetics can be exploited in diverse situations such as low-Reynolds fluid mixers [16], electrokinetic batteries [17], microfluidic circuitry [18], and salinity-based energyharvesting devices $[19,20]$ as well as biologically relevant systems such as transpiration in plants [15] or cortical bone fluid flows [14].

In order to characterize this electrokinetic transport regime, we will consider a symmetric, $z-z$ electrolyte solution, in contact with a reservoir of ionic strength $\rho_{0} z^{2}$, and filling a varying-section channel of length $L$ and halfaperture $h(x)=h_{0}-h_{1} \cos (2 \pi x / L)$ and whose walls, flat along the $z$ direction, have either a constant surface charge $\sigma$ or a constant electrostatic potential $\zeta$. The geometric impact of the channel corrugation on the electrokinetics of the liquid can be quantified in terms of the entropic barrier $\Delta S=\ln \left(h_{0}+h_{1}\right) /\left(h_{0}-h_{1}\right)$ [21]. In this highly confined geometry, we consider that the channel aperture varies smoothly, $\partial_{x} h(x) \ll 1$. This regime, where $\partial_{x}^{2} \ll \partial_{y}^{2}$ allows us to take advantage of the lubrication approximation [4] and reduce both the Poisson-Boltzmann equation governing the electrostatic field and the Stokes equation governing the low-Reynolds number fluid flow to 1D differential equations, as described in the Supplemental Material [22]. In this regime, we can disregard the dependence of the pressure on the transverse location, $P(x, y)=P(x)$, and assume that co- and counterions attain their equilibrium profile along the transverse direction, $y$, leading to a charge density

$$
q(x, y)=z e \rho_{+}(x) e^{-\beta z e \phi(x, y)}-z e \rho_{-}(x) e^{\beta z e \phi(x, y)},
$$

where $\beta^{-1}=k_{B} T$ corresponds to the inverse thermal energy, $e$ stands for the elementary charge, $z$ for the valence of the electrolyte, and $\phi(x, y)$ is the electrostatic potential. The varying channel aperture induces a variation of the overall ionic density along the channel, quantified by the amplitudes, $\rho_{ \pm}(x)$. Within the linearized Debye-Hückel regime, the electrostatic potential in the channel reads

$$
\phi(x, y)=\frac{\Lambda}{\kappa^{2}} \cosh (\kappa y)+\frac{z e}{\epsilon \kappa^{2}}\left[\rho_{+}(x)-\rho_{-}(x)\right],
$$

where $\kappa=\sqrt{4 \pi \ell_{B} z^{2}\left[\rho_{+}(x)+\rho_{-}(x)\right]}$ is the local inverse Debye length for an electrolyte of valence $z, \ell_{B}=\beta e^{2} / 4 \pi \epsilon$ stands for the Bjerrum length for an electrolyte with dielectric constant $\epsilon$, and $\Lambda$ is determined by the boundary condition on the channel walls [23].

The electrolyte dynamics is quite sensitive to the boundary conditions on the channel walls (either conducting or insulating) as well as to the nature of the external forcing (pressure or electric field; a detailed discussion can be found in the Supplemental Material [22]). We will focus on the electro-osmosis in a channel with insulating corrugated walls, when the induced dependence of the local electrostatic field along the channel is subdominant. As described in the Supplemental Material [22], imposing constant mass $J_{\rho}$, charge $J_{q}$, and solvent fluxes along the channel provide the steady-state charge density $\rho_{ \pm}$, pressure $P(x)$, and velocity $v(x)$ profiles [cf. Eqs. (2)-(6) in the Supplemental Material [22]]. The variable channel aperture introduces geometry-dependent constrictions and induces an inhomogeneous pressure gradient that controls the electrokinetic response. In equilibrium, the deviations of the ionic density from the magnitude corresponding to a uniform channel are negligible, $\rho_{+}^{\text {(eq) }}=\rho_{0} / 2$ [24].

In this regime, due to the channel smooth spatial corrugation, $\partial_{x} h(x) \ll 1$, the electrolyte response is dominated by the local pressure drop, while the contribution from the corrugation-induced corrections to the electrostatic potential is negligible. As a result, the transport and dynamics of electro- and pressure-driven electrolytes display qualitative and quantitative differences. On the contrary, for strongly corrugated channels $\partial_{x} h(x) \gg 1$, we expect the corrections in the ion density to overwhelm the local geometrically induced pressure gradients leading to a complementary scenario, beyond the perturbative approach described. Accordingly, we can identify two corrugationinduced electrokinetic regimes: when $\partial_{x} h(x) \ll 1$, ion densities are well captured by Eq. (1); i.e., the dynamics of the ion density is well captured by the concept of entropic barriers $[21,25,26]$. For $\partial_{x} h(x) \gg 1$, the factorization in Eq. (1) breaks down and the transverse ion distribution gets more involved.

Figure 1(b) displays the velocity profile of the electrolyte to leading order in the applied voltage drop, $\Delta V$ (corresponding to an applied uniform electric field, $E_{x}=$ $-\Delta V / L)$ along the channel when the variable channel section does not distort significantly the equilibrium electrostatic potential, $\phi_{0}$. Interestingly, in a corrugated channel characterized by a large entropic barrier, the electro-osmotic velocity in the central region of the channel flows against the mean fluid flow, $Q$. This velocity inversion appears due to the mismatch between the electro-osmotic fluid profile and the confinement-induced Poiseuille flow and grows with the geometric mismatch, or entropy barrier $\Delta S$, as displayed in Fig. 1(c). In particular, Fig. 1(d) shows that velocity reversal is magnified and reaches a significant magnitude when $\kappa^{-1}$ is comparable to the typical channel width, highlighting that entropic electrokinetic transport emerges from a competition between the reorganization in the charge profiles due to the geometrical confinement and its impact in the electroosmotic and pressure-driven flows characteristic of the 
entropic electrokinetic regime. Although we have focused on the linearized electrostatic regime to emphasize the origin of geometrically controlled electrolyte transport, the geometric restrictions induced by corrugated channels are robust and will affect electrokinetics also in strongly coupled [27] and strongly forced electrolytes [28]. Entropic electrokinetics, relevant to promote particle mixing at the micro- and nanoscale as well as for controlling electric currents, is more prominent for insulating walls [29] and vanishes for pressure-driven flows [30].

The electrokinetic flows induced by corrugated charged channels have a strong effect on the dynamics of suspended tracers. Tracers quantified in terms of their local concentration $C(x, y, t)$ will diffuse (with diffusion coefficient $D_{\text {tr }}$, will be advected according to the fluid velocity $v_{x}(x, y)$, and will be forced by the local electric field depending on the tracer charge. Following the FickJacobs approximation $[25,26,31]$ that has been shown to properly capture the dynamics of suspended tracers in corrugated channels [26] when $\partial_{x} h(x) \ll 1$, we can assume that tracers retain their equilibrium distribution along the transverse direction, and factorize $C(x, y, t)=$ $p(x, t) g(y \mid x)$, with

$$
g(y \mid x)=\frac{e^{-\beta U(x, y)}}{e^{-\beta A(x)}}, \quad e^{-\beta A(x)}=\int_{-\infty}^{\infty} e^{-\beta U(x, y)} d y
$$

in terms of the effective potential

$$
U(x, y)= \begin{cases}Z e\left(\phi(x, y)-x \tilde{E}_{x, 0}\right) & y \in[-h(x), h(x)] \\ \infty & \text { otherwise }\end{cases}
$$

being $Z$ the tracer valency and $p(x, t)$ the probability of finding a tracer at position $x$ at time $t$. For dilute tracers, we can disregard tracer-tracer interactions and tracer-induced corrections in the electrostatic fields and pressure gradient. Accordingly, tracer dynamics can be expressed in terms of an effective, $1 D$ convection-diffusion equation

$$
\frac{\partial}{\partial t} p=\frac{\partial}{\partial x}\left[\left(\frac{\partial}{\partial x} D_{\mathrm{tr}} \beta A(x)-\left\langle v_{x}\right\rangle_{y}\right) p+D_{\mathrm{tr}} \frac{\partial}{\partial x} p\right],
$$

where $\left\langle v_{x}\right\rangle_{y}=1 /(2 h(x)) \int_{-h(x)}^{h(x)} v(x, y) e^{-\beta U(x, y)} d y$ stands for the average velocity over the channel section. Equation (4) already shows that the tracer dynamics is governed by the average velocity field $\left\langle v_{x}\right\rangle_{y}$ and by the entropic barrier $A(x)$ that according to Eq. (3) has the shape of the equilibrium tracer free energy.

Figure 2 shows that tracer velocities, generically, decrease due to the entropic barriers induced by channel corrugation both for electrically and pressure-driven electrolytes for insulating walls [32]. In electro-osmosis in a flat channel, $\Delta S=0$, negatively charged tracers (closer to the positively charged surface) benefit from the forcing imposed by the external field and move faster than both positive and neutral ones [33]. Figure 2(a) shows that channel corrugation generically decreases asymmetrically the electro-osmotically induced tracer velocities. Increasing $\Delta S$, the fluid velocity develops a region of counterflow (as seen in Fig. 1) leading to a reduction of the convective contribution to the tracer motion with respect to the electrostatic driving, which affects different tracers depending on their charge. Hence, the corrugation, by modifying the velocity profile, acts as a selector that can enhance or reduce the convection of tracers depleted from the walls modulating the magnitude (and sign) of the tracer flux velocity. This geometrically induced tuning, characteristic of the entropic electrokinetic regime, can be quantified in terms of the competition between the characteristic tracer electrophoretic velocity due to the applied electric field $u_{e}=Z e \mu_{\mathrm{tr}} \Delta V_{0} / L$ involving the tracer mobility $\mu_{\mathrm{tr}}=\beta D_{\mathrm{tr}}$, and the typical electro-osmotic velocity the applied field induces in the solvent $u_{o}=Q / h_{0}$ that will tend to carry the tracers along. The inset of Fig. 2(a) shows the ratio $u_{e} / u_{o}$ as a function of the channel corrugation, $\Delta S$, and quantifies the impact that the entropic restrictions induced by the
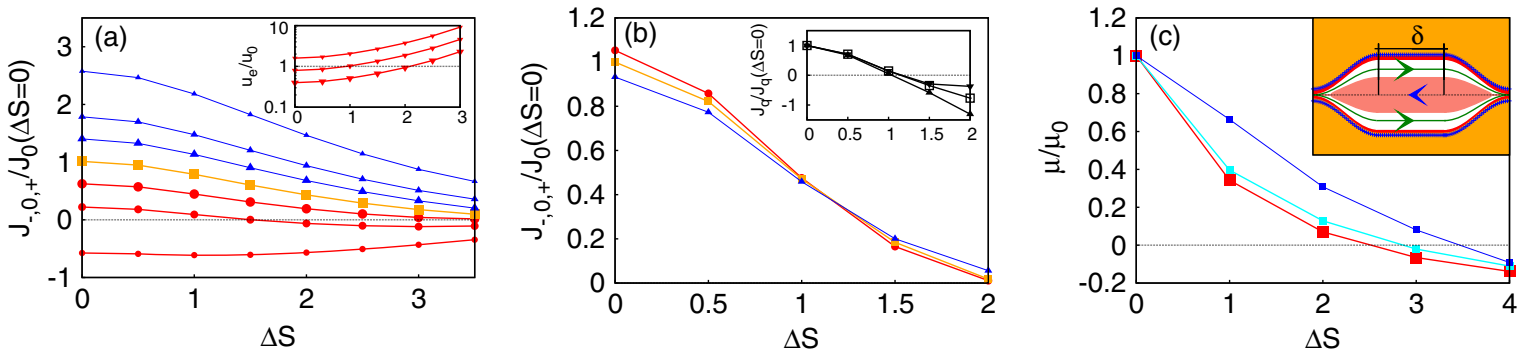

FIG. 2 (color online). Tracer flux in a varying-section channel as a function of the entropy barrier, $\Delta S$. (a) Positive (red dots), neutral (orange squares), or negative (blue triangles) tracer flux due to external electric field $\beta z e \Delta V=0.01$ characterized by $\kappa h_{0}=20$ for different tracer radii: $R / L=0.001,0.002,0.004$ where bigger points stand for bigger radii. Inset: Ratio between the external electrostatic and the convective force for the same data as in the main figure. (b) Positive (red dots), neutral (orange squares), or negative (blue triangles) tracer flux due to external pressure drop $\beta \Delta P h_{0}^{2}=0.001$ for tracer radius $R / L=0.004$. Inset: electric current $J_{q}$ normalized by its value at $\Delta S=0$ with $R / L=0.001,0.002,0.004$ for upper triangles, open squares, and lower triangles, respectively. (c) Mobility, $\mu$, of neutral tracers confined to the center of a corrugated channel, normalized by the mobility for a flat channel, $\mu_{0}$, as a function of $\Delta S$ for $R / L=10^{-4}$ and $\delta=1,2,3$ where bigger points stand for larger values of $\delta$. 
inhomogeneous channel section have in diminishing the effective drag induced by the electro-osmotic flow. For very high values of $\Delta S$, the convective driving is reduced by the geometrically enhanced dissipation leading to a reduction of the net fluid flux, $Q$. Moreover, the electrostatic driving is affected by the geometrically induced electrostatic barrier that forms, due to curvature, at the channel bottlenecks.

Tracers of radius $R$, due to their finite size, experience an effective, size-dependent corrugation, $\Delta S^{\prime}=\ln \left(h_{0}-R+\right.$ $\left.h_{1}\right) /\left(h_{0}-R-h_{1}\right)$, larger than the one affecting the electrolyte $\left(\Delta S^{\prime}>\Delta S\right)$. The restricted section sampled by the tracers, together with the velocity inversion in the channel center for electro-osmotic flows, as shown in Fig. 1, enhances mixing. Therefore, this additional geometric constraint leads to a complementary mechanism of geometric, or entropic origin, to control tracer segregation or tracer mixing at low-Reynolds numbers according to tracer size and/or charge, as shown in Fig. 2(a).

Figure 2(b) shows that for pressure-driven flows, channel corrugation also decreases the tracer velocity but does not lead to tracer inversion. Tracers attracted to the channel walls are more reactive to channel corrugations and experience a faster decrease of their velocity increasing $\Delta S$. The asymmetry in the sensitivity of positive and negative tracers to channel spatial inhomogeneities leads to an inversion in the tracer electric current, as shown in the inset of Fig. 2(b). This inversion emerges because the electrostatic potential is more susceptible to corrugation in the center of the channel, hence, having a stronger impact on tracers repelled by the channel walls. This is an experimentally detectable phenomenon because the signal-to-noise ratio captured by the tracer Péclet number $\mathrm{Pe}_{\mathrm{tr}}=u_{0} h_{0} / D_{\mathrm{tr}}$ can be of the order $\sim 1 / 10$ for $\Delta S \sim 3$. For example, this confinement-controlled current inversion will efficiently separate nanometer-size particles with charge $\pm e$ on length scales of the order of $\sim 10 \mu \mathrm{m}$ with micron-size average amplitude channels and Debye length $\kappa^{-1} \sim 5 \mathrm{~nm}$.

Local velocity inversion allows for negative mobility, where tracers displace on average against the net driving force $[34,35]$. This phenomenon is more clearly appreciated for neutral tracers, for which we can gain more insight because local electric forces do not compete with flowinduced drag. Tracers localized to the channel central section due the direct action of an external force (e.g., through optical trapping) will move against the mean electrolyte velocity when local flow inversion develops. Since the regions of flow inversion are compact and do not extend into the bottleneck regions, mobility inversion requires tracers to diffuse over the narrow region channel against the local flow to jump to the previous confined environment. As a result, the mobility rapidly decreases with the size of the inverse fluid flow region, as shown in Fig. 2(c), and can invert the sign for larger corrugations.
The variable channel corrugation modifies the tracer transverse probability distribution along the channel and enhances the role played by the central counterflux leading to velocity inversion. Hence, corrugation induces a new kind of absolute negative mobility that does not require disorder, characterized by two different regimes. In the diffusive regime, $\mathrm{Pe}_{\mathrm{tr}} \ll 1$, the time required to displace along the central part of the channel due to the reversed flux, $\tau_{\mathrm{cnv}}$, is the rate-limiting process, whereas in the convective regime, $\mathrm{Pe}_{\mathrm{tr}} \gtrsim 1$, the diffusion against the low in the channel bottlenecks, $\tau_{\text {diff }}$, becomes the limiting process. Therefore, modifying the shape of the channel offers a means to control the magnitude of the tracer negative mobility. For example, tuning the size, $\delta$, of the central region of the channel, it is possible to enlarge the relative relevance between $\tau_{\text {diff }}$ and $\tau_{\text {conv }}$ and, therefore, enhance the negative mobility, as shown in Fig. 2(c).

We have developed a flexible, theoretical framework that has allowed us to capture the essential dynamic properties of entropically induced electrokinetic flow in a corrugated channel when the Debye length is comparable to the channel section. We have found that electrokinetics in a spatially varying channel section allows for a very versatile and sensitive regulation of the fluxes of both electrolytes and tracers. We have clarified the relevance of confinement for a $z-z$ electrolyte driven by a pressure gradient or an electric potential drop and have shown that the induced electrokinetic flows lead to a variety of new phenomena, such as tracer separation for electro-osmosis, current inversion for pressure-driven fluxes, and negative mobility for optically trapped neutral tracers in an electro-osmotic flow. Entropically controlled electrokinetics can be achieved experimentally in a variety of conditions. For example, ion transport across nanometric porous media or the diffusion of radioactive ions in containers takes place along nanometric-size matrix [36], where the Debye length is naturally of the same size. Dilute electrolytes can develop micrometric-size Debye lengths, opening the possibility to study entropic electrokinetics of macromolecules, proteins, or aggregates in microfluidic devices. Finally, since channels with controllable corrugation have just been realized [37], entropic electrokinetics can provide an alternative way to the develop nano- and microfluidic devices.

We acknowledge the Dirección General de Investigación (Spain) and DURSI for financial support under Projects No. FIS 2011-22603 and No. 2009SGR-634, respectively. J.M.R. and I.P. acknowledge financial support from Generalitat de Catalunya under program Icrea Acadèmia.

* Corresponding author. paolomalgaretti@ffn.ub.es

[1] L. Bocquet and E. Charlaix, Chem. Soc. Rev. 39, 1073 (2010). 
[2] B. Alberts, A. Johnson, J. Lewis, M. Raff, K. Roberts, and P. Walter, Molecular Biology of the Cell (Garland Science, Oxford, 2007).

[3] R. Karnik, C. Duan, K. Castelino, and H. Daiguji, Nano Lett. 7, 547 (2007).

[4] S. Y. Park, C. J. Russo, D. Branton, and H. A. Stone, J. Colloid Interface Sci. 297, 832 (2006).

[5] A. Siria, P. Poncharal, R. Biance1, A.-L. Fulcrand, X. Blase, S. T. Purcell, and L. Bocquet, Nature (London) 494, 455 (2013).

[6] I. D. Kosinska, I. Goychuk, M. Kostur, G. Schmid, and P. Hänggi, Phys. Rev. E 77, 031131 (2008).

[7] S. Ghosal, J. Fluid Mech. 459, 103 (2002).

[8] K. Dorfman, Phys. Fluids 20, 037102 (2008).

[9] A. E. Malevich, V. Mityushev, and P. M. Adler, J. Colloid Interface Sci. 345, 72 (2010).

[10] D. Reguera, G. Schmid, P. S. Burada, J. M. Rubí, P. Reimann, and P. Hänggi, Phys. Rev. Lett. 96, 130603 (2006).

[11] U. M. B. Marconi, S. Melchionna, and I. Pagonabarraga, J. Chem. Phys. 138, 244107 (2013).

[12] S. Martens, A. V. Straube, G. Schmid, L. Schimansky-Geier, and P. Hänggi, Phys. Rev. Lett. 110, 010601 (2013).

[13] I. Pagonabarraga, B. Rotenberg, and D. Frenkel, Phys. Chem. Chem. Phys. 12, 9566 (2010).

[14] T. Lemaire, S. Naili, and A. Remond, Biomechan. Model Mechanobiol. 5, 39 (2005).

[15] T. Wheeler and A. Stroock, Nature (London) 455, 208 (2008).

[16] A. D. Stroock, S. K. W. Dertinger, A. Ajdari, I. Mezic, H. A. Stone, and G. M. Whitesides, Science 295, 647 (2010).

[17] J. Yang, F. Lu, L. W. Kostiuk, and D. Y. Kwok, J. Micromech. Microeng. 13, 963 (2003).

[18] C. B. Picallo, S. Gravelle, L. Joly, E. Charlaix, and L. Bocquet, Phys. Rev. Lett. 111, 244501 (2013).

[19] D. Brogioli, Phys. Rev. Lett. 103, 058501 (2009).

[20] N. Boon and R. van Roij, Mol. Phys. 109, 1229 (2011).

[21] P. Malgaretti, I. Pagonabarraga, and J. M. Rubi, J. Chem. Phys. 138, 194906 (2013).

[22] See Supplemental Material at http://link.aps.org/ supplemental/10.1103/PhysRevLett.113.128301 for more detail the derivation of the effective expressions that quantify the transport of electrolyte along corrugated channels. It also provides data of flow reversal for conducting solid walls.
[23] For insulating channel walls with surface charge $\sigma$, local electroneutrality is always preserved in the lubrication regime. Although we disregard variation of the electric field at the confining wall due to the channel corrugation (see, e.g., Ref. [38]), its influence is of higher order than the results we will analyze.

[24] These results shed light on the corrugation-induced correction at equilibrium, stating that up to second order in the surface charge, there is no relevant correction to the homogeneous profile $\rho_{ \pm}^{(\mathrm{eq})}=\rho_{0} / 2$.

[25] R. Zwanzig, J. Phys. Chem. 96, 3926 (1992).

[26] D. Reguera and J. M. Rubi, Phys. Rev. E 64, 061106 (2001).

[27] Since the confined electrokinetic regime relies on the flow inversion stemming from incompressibility, we expect it to be robust for strongly coupled electrolytes.

[28] The corrections due to the imposed electric field arise through the deformations it induces in the ionic distribution. The magnitude of the electric field parallel to the channel is of order $F_{\text {ext }} \simeq z e E \partial_{x} h$, while the electric field due to the charged wall has a typical magnitude $F_{D b y} \simeq z e \phi_{0} \kappa$. Therefore, the distortion of the charge profile along the channel is negligible when $E \ll \kappa \phi_{0} / \partial_{x} h$, which is easily achieved in narrow or slowly varying channels, $\partial_{x} h \ll 1$.

[29] For conducting walls, velocity reversal develops for strong corrugations, as shown in the Supplemental Material [22].

[30] For pressure-driven flows, the matching between the driven and the induced flux affects more mildly the overall flow not giving rise to flow inversion in the center of the channel.

[31] P. Kalinay and J. K. Percus, Phys. Rev. E 78, 021103 (2008).

[32] Analogous results are obtained for conducting walls, although of smaller magnitude (everything else being equal).

[33] B. Rotenberg, I. Pagonabarraga, and D. Frenkel, Europhys. Lett. 83, 34004 (2008).

[34] R. Eichhorn, J. Regtmeier, and P. Reimann, Soft Matter 6, 1858 (2010).

[35] D. Speer, R. Eichhorn, M. Evstigneev, and P. Reimann, Phys. Rev. E 85, 061132 (2012).

[36] A. Bertron, N. Jacquemet, B. Erable, C. Sablayrolles, G. Escadeillas, and A. Albrecht, Nucl. Eng. Des. 268, 51 (2014).

[37] D. Chakraborty, J. R. Prakash, J. Friend, and L. Yeo, Phys. Fluids 24, 102002 (2012).

[38] D. Gillespie, W. Nonner, and R. S. Eisenberg, J. Phys. Condens. Matter 14, 12129 (2002). 